\title{
Analysis of in vitro chemoresponse assays in endometrioid endometrial adenocarcinoma: an observational ancillary analysis
}

\author{
Brittany A. Davidson ${ }^{1 *}$, Jonathan Foote ${ }^{1}$, Stacey L. Brower ${ }^{2}$, Chunqiao Tian², Laura J. Havrilesky ${ }^{1}$ \\ and Angeles Alvarez Secord ${ }^{1}$
}

\begin{abstract}
Background: Chemotherapy plays a role in the treatment of endometrioid endometrial cancer (EEC); however, tumor grade may affect response. Our objective was to evaluate associations between tumor grade and in vitro chemoresponse.

Methods: We conducted an analysis of primary tumor samples from women with EEC undergoing in vitro chemoresponse testing. Results were classified as sensitive (S), intermediate (I), or resistant (R) to each drug tested. Correlations between tumor grade and response were examined.

Results: Data was collected from 159 patients: 28 with grade 1 (18\%), 52 with grade 2 (32\%), and 79 (50\%) with grade 3 tumors. Median age of patients was 62 (range 31-92). Most patients were Caucasian (83\%) with advanced disease (Stage III: 50.9\%; Stage IV: 13.2\%). Overall chemoresponse was similar across all grades. Fifty percent, 56 and $51 \%$ for grade 1, 2, and 3 tumors, respectively, demonstrated $S$ results to at least 1 agent. There was no association between grade and in vitro response to chemotherapy agents $(p>0.05)$ except a marginal association between grade and doxorubicin response $(p=0.08)$. Grade 1 and 2 cancers were more likely to demonstrate $R$ results for doxorubicin compared to grade 3 cancers (G1: $19 \%$ vs G2: $25 \%$ vs G3: 8\%; $p=0.08$ ). In a subset tested for all 7 agents, only one patient tumor was pan-R and 4 were pan-S.
\end{abstract}

Conclusions: Based on our data, grades 1-3 EEC have similar in vitro chemoresponse. These findings suggest that chemotherapy may be useful in advanced low grade EECs, but further clinical correlation is needed.

Keywords: Endometrial cancer, Chemosensitivity, Tumor grade, Endometrioid

\section{Background}

Endometrial cancer (EC) is the most common gynecologic malignancy, with nearly 55,000 new cases and more than 10,000 deaths predicted for 2015 [1]. While 5 year survival trends have improved for other gynecologic malignancies, the survival rate for patients diagnosed with EC between 2004 and 2010 is lower than that of patients diagnosed between 1975 and 1977 (83 vs. $87 \% p=<0.05)[1]$. Undoubtedly, many factors

\footnotetext{
* Correspondence: Brittany.davidson@duke.edu

'Division of Gynecologic Oncology, Duke University Medical Center, Duke

University, DUMC Box 3079, Durham, NC 27710, USA

Full list of author information is available at the end of the article
}

account for this trend; however it is yet unknown if chemoresistance plays an important role.

The mechanism underlying chemoresistance in EC is uncertain, however current dogma suggests that low grade Type I endometrioid endometrial cancers (EEC) are less likely to respond to chemotherapy. Type I tumors represent the majority of sporadic EC, characterized predominantly by endometrioid histology and expression of estrogen and/or progesterone receptors [2]. In contrast, Type II EC is less common and often of serous or clear cell histology, arising in atrophic endometrium, rather than estrogen excess [3]. There is 
conflicting data regarding chemotherapy response and tumor grade and histology in endometrial cancers.

Response rate (RR) to chemotherapy was not significantly different between endometrioid (44\%), clear cell (32\%) and serous tumors (44\%) in a pooled analysis of patients with advanced or recurrent EC treated on 1 of 4 GOG trials (GOG 107, GOG 139, GOG 163, GOG 177) (clear cell $p=0.13$; serous $p=0.99$ ) [4]. In a subgroup analysis of over 600 patients with endometrioid histology alone enrolled in these same 4 GOG trials, grade 3 tumors had an estimated odds of response of approximately 1.5 times that of grade 1 tumors, although these results were not statistically significant $(p=0.09)$ [4]. However, these two analyses were based on a retrospective assessment of investigator-determined response which may be prone to subjective assessment and error.

We previously explored the association between tumor grade and cytotoxic treatment response in patients with advanced or recurrent EEC $(N=91)$. Contrary to expectations, grade 2 cancers were more likely to respond to all types of chemotherapy ( 72 vs $43 \% p=0.02$ ) and to carboplatin/paclitaxel doublets (72 vs $41 \% \quad p=0.02$ ) compared to grade 3 cancers [5]. However, this study was limited by lack of central pathology review, small sample size, and paucity of patients with grade 1 ECC.

Given the contradictory data and limitations of prior studies, we explored in vitro chemoresponse profiles to obtain insight into the relationship between chemotherapeutic anti-tumor activity and grade in EEC specimens from women enrolled in observational studies.

\section{Methods}

The study population included women with endometrioid endometrial cancer whose primary cancer specimens were submitted for in vitro chemoresponse assay testing on prospectively-accrued observational studies between 2006 and 2010. These were longitudinal, observational multi-center studies examining the outcomes associated with chemosensitivity assays in women with gynecologic malignancies. Participants had not received chemotherapy prior to specimen collection. Tumor grades were assigned by the institutions submitting the specimens for testing. Assays were conducted for up to 7 cytotoxic agents including carboplatin, cisplatin, doxorubicin, paclitaxel, docetaxel, gemcitabine, and topotecan.

Details regarding the particular chemoresponse assay used in this study (ChemoFx ${ }^{\circ}$, Helomics Corporation, Pittsburgh, PA) have been described elsewhere [6, 7]. Assay preparation included an immunocytochemistry step to aid in the confirmation that cells were of epithelial, rather than stromal, origin. All cultures required a majority of epithelial cells to proceed to chemoresponse testing. A board-certified pathologist assessed cell morphology.
Inhibition of tumor growth was measured at several serially-diluted concentrations of each cytotoxic agent tested. For each drug, the area under the dose-response curve (AUC) was calculated. Greater sensitivity to the therapy tested was indicated by a smaller AUC. Using established criteria, tumor chemoresponse was classified using the in vitro AUC score into one of three categories: sensitive (S), intermediate sensitive (I), or resistant (R). The in vitro tumor response rate (RR) for each agent was then defined as the proportion of patients with tumors testing either $\mathrm{S}$ or I for that agent.

The primary endpoint of this ancillary study was to assess the association between tumor grade and in vitro chemoresponse assay results in EEC. Patient demographics were also collected, including age and stage at diagnosis. Correlations of tumor grade with assay results were examined using Cochran-Armitage test for trend using SAS version 9.4 (SAS Institute, Cary, NC).

\section{Results}

A total of 159 patients were included for this analysis. Twenty-eight patients had grade 1 (18\%), 52 had grade 2 $(32 \%)$, and $79(50 \%)$ had grade 3 tumors. The median age of patients was 62 (range 31-92). Most patients were Caucasian (83\%) and had advanced stage disease (Stage III: 50.9\%; Stage IV: 13.2\%) at diagnosis [Table 1].

As mentioned previously, the chemotherapeutic agents tested in this assay included carboplatin, cisplatin, doxorubicin, and paclitaxel, which are commonly used in EC. In addition, other cytotoxics incorporated in the panel

Table 1 Patient Characteristics by Tumor Grade

\begin{tabular}{|c|c|c|c|c|}
\hline & $\begin{array}{l}\text { Grade } 1 \\
(n=28)\end{array}$ & $\begin{array}{l}\text { Grade } 2 \\
(n=52)\end{array}$ & $\begin{array}{l}\text { Grade } 3 \\
(n=79)\end{array}$ & $\begin{array}{l}\text { Total } \\
(n=159)\end{array}$ \\
\hline & No. (\%) & No. (\%) & No. (\%) & No. (\%) \\
\hline \multicolumn{5}{|l|}{ Age (years) } \\
\hline Median (Range) & $63.5(42-92)$ & $62(32-87)$ & $62(31-89)$ & $62(31-92)$ \\
\hline$<50$ & $2(7.1)$ & $8(15.4)$ & $9(11.4)$ & 19 (11.9) \\
\hline $50-64$ & $13(46.4)$ & $22(42.3)$ & $36(45.6)$ & $71(44.7)$ \\
\hline $65-74$ & 8 (28.6) & $14(26.9)$ & $24(30.4)$ & $46(28.9)$ \\
\hline$\geq 75$ & $5(17.9)$ & $8(15.4)$ & $10(12.7)$ & $23(14.5)$ \\
\hline \multicolumn{5}{|l|}{ Race } \\
\hline White & $24(85.7)$ & $45(86.5)$ & $63(79.7)$ & $132(83)$ \\
\hline Black & $1(3.6)$ & $4(7.7)$ & $12(15.2)$ & $17(10.7)$ \\
\hline Other & $3(10.7)$ & $3(5.8)$ & $4(5.1)$ & $10(6.3)$ \\
\hline \multicolumn{5}{|l|}{ FIGO Stage } \\
\hline 1 & $11(39.3)$ & $8(15.4)$ & $21(26.6)$ & $40(25.2)$ \\
\hline$\|$ & $0(0.0)$ & $8(15.4)$ & $7(8.9)$ & $15(9.4)$ \\
\hline III & $15(53.6)$ & $28(53.8)$ & $38(48.1)$ & $81(50.9)$ \\
\hline IV & $2(7.1)$ & $6(11.5)$ & $13(16.5)$ & $21(13.2)$ \\
\hline Unknown & $0(0.0)$ & $2(3.8)$ & $0(0.0)$ & $2(1.3)$ \\
\hline
\end{tabular}


were evaluated including docetaxel, gemcitabine, and topotecan. Not all patients had their tumors tested for all 7 cytotoxic agents. Assay results were available for the following cytotoxic agents stratified by grade 1,2 , and 3: carboplatin (25/28[89\%], 41/52[79\%], 66/79[84\%]), paclitaxel (24/28[86\%], 40/52[77\%], 65/79[82\%]), doxorubicin (27/ 28[96\%], 48/52[92\%], 77/79[97\%]), cisplatin (24/28[86\%], 43/52[83\%], 67/79[85\%]), docetaxel (19/28[68\%], 35/ $52[67 \%], \quad 61 / 79[77 \%])$, gemcitabine (14/28[50\%], 26/ $52[50 \%], 45 / 79[56 \%])$, and topotecan (22/28[71\%], 41/ 52[79\%], 61/79[77\%]) [Table 2].

The number of $\mathrm{R}$ results, defined as responses that were neither S nor I, was similar across grades. Twenty seven percent, 21 , and $17 \%$ for grade 1,2 , and 3 tumors, respectively, demonstrated resistant chemoresponse results for

Table 2 In Vitro Tumor Responses to Seven Drugs

\begin{tabular}{|c|c|c|c|c|c|c|}
\hline \multicolumn{7}{|l|}{ Assay Result ${ }^{b}$} \\
\hline Drug & Tumor Grade & No. patients & $\mathrm{R}(\%)$ & I (\%) & S (\%) & $P$ value $^{a}$ \\
\hline \multirow[t]{4}{*}{ Carboplatin } & & & & & & .928 \\
\hline & 1 & 25 & 28.0 & 40.0 & 32.0 & \\
\hline & 2 & 41 & 24.4 & 39.0 & 36.6 & \\
\hline & 3 & 66 & 19.7 & 53.0 & 27.3 & \\
\hline \multirow[t]{4}{*}{ Cisplatin } & & & & & & .322 \\
\hline & 1 & 24 & 29.2 & 41.7 & 29.2 & \\
\hline & 2 & 43 & 18.6 & 55.8 & 25.6 & \\
\hline & 3 & 67 & 13.4 & 58.2 & 28.4 & \\
\hline \multirow[t]{4}{*}{ Docetaxel } & & & & & & .812 \\
\hline & 1 & 19 & 26.3 & 42.1 & 31.6 & \\
\hline & 2 & 35 & 8.6 & 54.3 & 37.1 & \\
\hline & 3 & 61 & 18.0 & 47.5 & 34.4 & \\
\hline \multirow[t]{4}{*}{ Doxorubicin } & & & & & & .080 \\
\hline & 1 & 27 & 18.5 & 55.6 & 25.9 & \\
\hline & 2 & 48 & 25.0 & 56.3 & 18.8 & \\
\hline & 3 & 77 & 7.8 & 61.0 & 32.2 & \\
\hline \multirow[t]{4}{*}{ Gemcitabine } & & & & & & .238 \\
\hline & 1 & 14 & 28.6 & 28.6 & 42.9 & \\
\hline & 2 & 26 & 30.8 & 23.1 & 46.2 & \\
\hline & 3 & 45 & 22.2 & 62.2 & 15.6 & \\
\hline \multirow[t]{4}{*}{ Paclitaxel } & & & & & & .670 \\
\hline & 1 & 24 & 33.3 & 33.3 & 33.3 & \\
\hline & 2 & 40 & 15.0 & 37.5 & 47.5 & \\
\hline & 3 & 65 & 15.4 & 53.9 & 30.8 & \\
\hline \multirow[t]{4}{*}{ Topotecan } & & & & & & .861 \\
\hline & 1 & 22 & 27.3 & 45.5 & 27.3 & \\
\hline & 2 & 41 & 24.4 & 48.8 & 26.8 & \\
\hline & 3 & 61 & 23.0 & 50.8 & 26.2 & \\
\hline
\end{tabular}

${ }^{\mathrm{a}}$ Correlation of tumor grade with assay result examined by Cochran-Armitage test

${ }^{\mathrm{b}} R$ resistant, $I$ intermediately sensitive, $S$ sensitive any of the agents tested. There was no association between tumor grade and in vitro response to various chemotherapy agents $(p>0.05)$ other than a marginal association between grade and response to doxorubicin $(p=0.08)$ (Table 2). Specifically, grade 1 and 2 cancers were more likely to demonstrate $\mathrm{R}$ assay results for doxorubicin compared to grade 3 cancers (G1: $18 \%$ vs G2: $25 \%$ vs G3: $8 \%$; $p=0.08)$.

Ten of 28 (36\%) grade 1, 21/52 (38\%) grade 2, and 33/ 79 (42\%) grade 3 tumors were tested for all 7 cytotoxic agents (Fig. 1). Of those grade 1 tumors tested for all 7 cytotoxic agents, 6/10 (60\%) were pan-sensitive (defined as $\mathrm{S}+\mathrm{I}$ ), and none were pan-resistant. Of the 21 grade 2 tumors tested for all 7 agents, 10 (47.6\%) were pansensitive and 1 (5\%) pan-resistant. Similarly, 19 of 33 (57.6\%) grade 3 tumors were pan-sensitive while none were pan-resistant. When examining the entire cohort of tumors, 14/ 28 (50\%) grade 1 tumors exhibited a sensitive ( $\mathrm{S}$ only) chemoresponse assay to at least 1 agent. Similarly, 29/52 (56\%) of grade 2 tumors and 40/79 (51\%) grade 3 tumors also showed in vitro sensitivity to at least one cytotoxic agent. If $\mathrm{S}+\mathrm{I}$ responses are considered, these RRs increase to 73,79 and $83 \%$ for grades 1,2 and 3 tumors, respectively.

\section{Discussion}

Our data suggests there is no difference in in vitro chemoresponse among EECs of various grades. When stratified by tumor grade, RRs were similar for 7 chemotherapeutic agents with the exception of doxorubicin, where grade 3 tumors exhibited a non-significant increased RR $(92 \%(\mathrm{~S}+\mathrm{I}))$ compared to grade $1(81 \%)$ and grade $2(75 \%)$ tumors. Overall our results are similar to the clinical findings reported by McMeekin and colleagues examining the relationship between tumor histology and chemotherapeutic response in 4 GOG endometrial cancer trials (GOG\#107, GOG\#139, GOG\#163, GOG\#177). To date, this GOG ancillary analysis is the largest study to examine the association between tumor grade and chemotherapy response in EEC [4]. These trials encompassed both advanced and recurrent EC treated with a variety of cytotoxic agents, including doxorubicin, a doxorubicin/cisplatin doublet, a doxorubicin/paclitaxel doublet, and a doxorubicin/ cisplatin/paclitaxel triplet administered in various intervals and doses [8-11]. The majority of patients included in these studies had also received prior radiation therapy. In a subgroup analysis of over 600 patients with endometrioid histology only, there was no difference in response of grade 3 versus grade 1 tumors ( $p=$ 0.09). Furthermore, tumor grade was not associated with progression free survival (PFS) or overall survival [4]. This may be due, in part, to the fact that patients had advanced or recurrent disease associated with a 


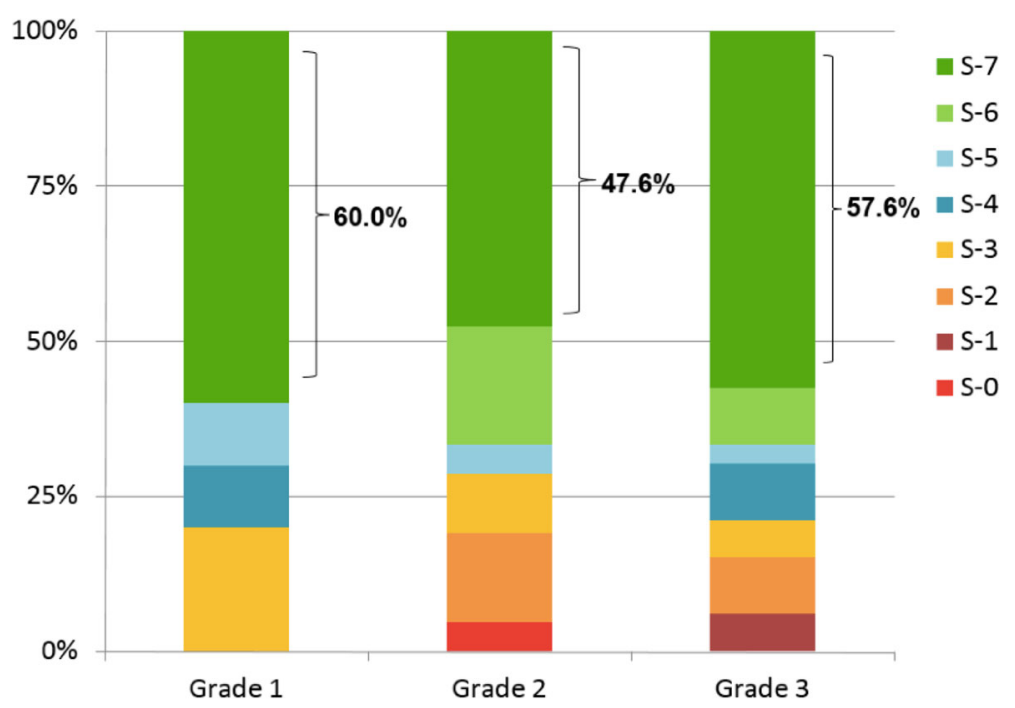

Fig. 1 Distribution of assay results across seven single agent treatments. Patients $(n=64)$ were categorized based on response to carboplatin, cisplatin, doxorubicin, gemcitabine, paclitaxel, docetaxel, and topotecan. Tumors were either resistant to all drugs (S0), or sensitive $(\mathrm{S}+\mathrm{I})$ from anywhere from one (S1) agent to all 7 agents (S7). Sixty percent of grade 1 tumors were pan-sensitive (S7) vs 47.6 and $57.6 \%$ of grades 2 and 3

poor prognosis, limiting the ability to detect differences in subgroups. While there was heterogeneity among these various studies, the strength of the ancillary analysis of the GOG trials is the uniform delivery of therapy in a prospective clinical trial setting, and pathologic confirmation of tumor grade and histology required for enrollment.

The correlation between in vitro chemoresponse \& tumor grade, as well as the correct definition of this response ( $\mathrm{S}+\mathrm{I}$ vs $\mathrm{S}$ ) in women with EC is unknown. We compared our in vitro chemotherapy response to the $\mathrm{RR}$ reported in the literature for combination carboplatin/paclitaxel (CT) or cisplatin/doxorubicin/paclitaxel (TAP), the current standard of care therapy for advanced or recurrent EC. In our study, RRs based on in vitro chemotherapy response defined as $\mathrm{S}+\mathrm{I}$, were noted for the following agents: carboplatin, 77\%; paclitaxel, $81 \%$; cisplatin, $82 \%$; and doxorubicin, $85 \%$. These in vitro chemotherapy RRs are nearly twice the RRs reported in the 4 GOG trials studied by McMeekin et al. [4] However, when only $S$ results are considered (carboplatin, $31 \%$; paclitaxel, $36 \%$; cisplatin, $28 \%$; doxorubicin: $26 \%$ ), our in vitro results are similar to those reported in several small phase II trials evaluating the use of single agents in chemo naïve patients with EC. For example, $20 \%$ of women with chemo naïve advanced or recurrent $\mathrm{EC}$ receiving single agent cisplatin achieved a response [12], compared to a $28 \%$ in vitro response in our analysis. Furthermore, our in vitro results were nearly identical to those seen with paclitaxel $(14.3 \%$ CR, 21.4\% PR) in GOG-86O and doxorubicin (RR 25\%) in GOG-107 [10, 13]. No in vitro doublet assays were performed for comparison with in vivo studies. Comparisons between the populations in these prior studies and our results are limited due to different dosing schedules as well as the in vitro vs. in vivo differences. However, the results suggest that in vitro studies may be applicable in predicting tumor response in vivo.

Data from epithelial ovarian cancer (EOC) specimens have compared in vitro assay response to clinical outcomes. Krivak and colleagues reported that in vitro assay resistance to carboplatin is associated with decreased PFS in women with advanced-stage EOC treated with platinum based therapy. Specifically, women whose tumor specimens demonstrated in vitro platinum resistance were at higher risk for disease progression compared to those with sensitive or intermediate sensitive assay results (median PFS: 11.8 vs 16.6 months, respectively, $P<.001$ ) [6]. In addition, our group compared in vitro assay response between Type I and Type II EOC and found that, despite the dogmatic belief that Type I EOC are chemoresistant, the majority (86\%) of Type I tumors were chemosensitive to at least one cytotoxic agent and $35.7 \%$ were pan-S to all 7 agents tested [14]. Multi-drug resistance was twice as likely in women with Type I EOC compared to Type II EOC (pan-R, 14.3 vs. $6.8 \%(p=0.268)$; pan-S, 35.7 vs. $51.2 \%(p=0.183))$, but did not reach statistical significance. Similarly, in our analysis in EC, $20 \%$ of grade 1 tumors were pansensitive. None of the grade $1 \mathrm{ECs}$ were resistant to the 7 agents included in the cytotoxic panel, indicating that chemotherapy may be useful in the treatment of grade 1 advanced or recurrent disease. These recent studies demonstrate the clinical validity and utility of in vitro 
chemotherapy assays to direct therapy. Continued evaluation could further support the role of this test in clinical practice, especially given the interest in precision and personalized medicine.

Understanding the intricacies of molecular differences in EC histologies may be fundamental in directing targeted therapies. Data from The Cancer Genome Atlas (TCGA) have identified molecular signatures that may account for heterogeneity in treatment response for endometrioid endometrial tumors. While only a small percentage of grade 1 and 2 EECs have genetic fingerprints similar to serous endometrial cancers, almost 25\% of grade 3 EECs possess signatures closely related to these more aggressive tumors [15]. This data supports the possibility of including grade 3 EECs with other Type II EECs. However, it is uncertain if these molecular signatures are associated with or can differentially predict response to chemotherapy in endometrioid cancers and Type II cancers. Additional analyses into molecular fingerprints of these malignancies have also helped to characterize pathways that may be involved in low grade, yet aggressive EEC in young women. While the PI3K pathway is a source of frequent mutations in EEC, differential expression of hotspot mutations have been noted in microsatellite stable vs. instable endometrial tumors [16]. As we learn more about the intricacies of the cancer genome, it is apparent that histology and tumor grade may not be the only factors that determine the behavior of these endometrial malignancies.

Limitations of this study include the lack of size equivalence between the tumor grade cohorts (namely, fewer tumors in the grade 1 cohort). In addition, not all tumors underwent chemosensitivity testing to all 7 agents as physicians could choose which drugs to submit, thus introducing a selection bias. Strengths of this study include the prospective collection of data that was routinely and comprehensively monitored, as well as the uniform preparation and performance of the chemosensitivity assay. Less than $20 \%$ of submitted samples fail the assay due to insufficient cell growth in culture or contamination. We were unable to assess the association between in vitro response results and clinical RR and survival outcomes due to limited clinical data.

\section{Conclusion}

Based on our results, there does not appear to be an association between tumor grade and in vitro chemoresponse assay results. Specifically low grade EECs are not more likely to have resistant assay results compared to higher grade EECs. In addition, 50\% of grade 1 EECs demonstrated in vitro sensitivity to at least one cytotoxic therapy, suggesting that chemotherapy may be useful in advanced low grade EECs. However, further clinical correlation is needed to assess assay sensitivity/resistance to in vivo response and clinical outcomes to determine if chemoresponse assays may be useful to direct therapy in women with endometrioid endometrial cancer.

\section{Abbreviations}

AUC: Area under the dose response curve; C/T: Carboplatin/paclitaxel;

EC: Endometrial cancer; EEC: Endometrioid endometrial cancer;

EOC: Epithelial ovarian cancer; I: Intermediate; R: Resistant; RR: Response Rate; S: Sensitive; TAP: Cisplatin/doxorubicin/paclitaxel

\section{Acknowledgements}

Not applicable

\section{Authors' contributions}

BD designed and interpreted the statistical analyses and is the lead author of the manuscript. JF was a major contributor in the writing of the manuscript. SB provided the raw data \& contributed to the statistical analysis. CT performed the majority of the statistical analyses. A.S. served as the primary mentor to $\mathrm{BD}$ on this research project and was a major contributor in writing the manuscript. L.H. served as the secondary mentor to BD and was a major contributor in writing the manuscript. All authors read and approved the final manuscript.

\section{Competing interests}

A.S. has served on Advisory Boards for Precision Therapeutics (now known has Helomics). The authors declare that they have no competing interests.

\section{Consent for publication \\ Not applicable.}

Ethics approval and consent to participate

Not applicable.

\section{Author details}

${ }^{1}$ Division of Gynecologic Oncology, Duke University Medical Center, Duke University, DUMC Box 3079, Durham, NC 27710, USA. ²Product Development, Helomics Corporation, Pittsburgh, PA, USA.

Received: 30 June 2016 Accepted: 15 November 2016

Published online: 01 December 2016

\section{References}

1. Siegel RL, Miller KD, Jemal A. Cancer statistics, 2015. CA Cancer J Clin. 2015; 65(1):5-29.

2. Dottino JA, Cliby WA, Myers ER, Bristow RE, Havrilesky LJ. Improving NCCN guideline-adherent care for ovarian cancer: value of an intervention. Gynecol Oncol. 2015.

3. Doll A, Abal M, Rigau M, Monge M, Gonzalez M, Demajo S, et al. Novel molecular profiles of endometrial cancer-new light through old windows. J Steroid Biochem Mol Biol. 2008;108(3-5):221-9.

4. McMeekin DS, Filiaci VL, Thigpen JT, Gallion HH, Fleming GF, Rodgers WH, et al. The relationship between histology and outcome in advanced and recurrent endometrial cancer patients participating in first-line chemotherapy trials: a gynecologic oncology group study. Gynecol Oncol. 2007;106(1):16-22

5. Davidson BFJ, Clarke LH, et al. Tumor grade and chemotherapy response in endometrioid endometrial cancer. 2015

6. Krivak TC, Lele S, Richard S, Secord AA, Leath 3rd CA, Brower SL, et al. A chemoresponse assay for prediction of platinum resistance in primary ovarian cancer. Am J Obstet Gynecol. 2014;211(1):68. e1-8.

7. Rutherford T, Orr Jr J, Grendys Jr E, Edwards R, Krivak TC, Holloway R, et al. A prospective study evaluating the clinical relevance of a chemoresponse assay for treatment of patients with persistent or recurrent ovarian cancer. Gynecol Oncol. 2013;131(2):362-7.

8. Fleming GF, Brunetto VL, Cella D, Look KY, Reid GC, Munkarah AR, et al. Phase III trial of doxorubicin plus cisplatin with or without paclitaxel plus filgrastim in advanced endometrial carcinoma: a gynecologic oncology group study. J Clin Oncol. 2004;22(11):2159-66.

9. Fleming GF, Filiaci VL, Bentley RC, Herzog T, Sorosky J, Vaccarello L, et al. Phase III randomized trial of doxorubicin + cisplatin versus doxorubicin + 
24-h paclitaxel + filgrastim in endometrial carcinoma: a gynecologic oncology group study. Ann Oncol. 2004;15(8):1173-8.

10. Thigpen JT, Brady MF, Homesley HD, Malfetano J, DuBeshter B, Burger RA, et al. Phase III trial of doxorubicin with or without cisplatin in advanced endometrial carcinoma: a gynecologic oncology group study. J Clin Oncol. 2004;22(19):3902-8.

11. Gallion HH, Brunetto VL, Cibull M, Lentz SS, Reid G, Soper JT, et al. Randomized phase III trial of standard timed doxorubicin plus cisplatin versus circadian timed doxorubicin plus cisplatin in stage III and IV or recurrent endometrial carcinoma: a gynecologic oncology group study. J Clin Oncol. 2003;21(20):3808-13.

12. Thigpen JT, Blessing JA, Homesley H, Creasman WT, Sutton G. Phase II trial of cisplatin as first-line chemotherapy in patients with advanced or recurrent endometrial carcinoma: a gynecologic oncology group study. Gynecol Oncol. 1989:33(1):68-70.

13. Ball HG, Blessing JA, Lentz SS, Mutch DG. A phase II trial of paclitaxel in patients with advanced or recurrent adenocarcinoma of the endometrium: a gynecologic oncology group study. Gynecol Oncol. 1996;62(2):278-81.

14. Previs R, Leath 3rd CA, Coleman RL, Herzog TJ, Krivak TC, Brower SL, et al. Evaluation of in vitro chemoresponse profiles in women with type I and type II epithelial ovarian cancers: an observational study ancillary analysis. Gynecol Oncol. 2015:138(2):267-71.

15. Cancer Genome Atlas Research N, Kandoth C, Schultz N, Cherniack AD, Akbani R, Liu Y, et al. Integrated genomic characterization of endometrial carcinoma. Nature. 2013;497(7447):67-73.

16 Marchio C, De Filippo MR, Ng CK, Piscuoglio S, Soslow RA, Reis-Filho JS, et al. PIKing the type and pattern of PI3K pathway mutations in endometrioid endometrial carcinomas. Gynecol Oncol. 2015;137(2):321-8.

\section{Submit your next manuscript to BioMed Central and we will help you at every step:}

- We accept pre-submission inquiries

- Our selector tool helps you to find the most relevant journal

- We provide round the clock customer support

- Convenient online submission

- Thorough peer review

- Inclusion in PubMed and all major indexing services

- Maximum visibility for your research

Submit your manuscript at www.biomedcentral.com/submit

C) Biomed Central 